\title{
Head and Neck Tuberculosis associated to Sarcoidosis : A Case Report.
}

\author{
Azza Mediouni ${ }^{1}$, Sabrine Najjar ${ }^{1}$, Seif Boukriba ${ }^{1}$, Beya Chelly ${ }^{1}$, houda chahed ${ }^{1}$, and Ghazi \\ Besbes $^{2}$ \\ ${ }^{1}$ University of Tunis El Manar Faculty of Medicine of Tunis \\ ${ }^{2}$ University of Tunis El Manar
}

September 25, 2021

\begin{abstract}
The distinction between tuberculosis and sarcoidosis presents sometimes a clinical challenge. Their sequential occurrence in the same patient is uncommon. We present the case of a 42-year-old female with a proven diagnosis of tuberculous lymphadenitis who has developed successively nasal tuberculosis and pulmonary and liver sarcoidosis.
\end{abstract}

Title of the article:Head and Neck Tuberculosis associated to Sarcoidosis: A Case Report.Contributors:Azza Mediouni; MD Affiliations: 1-University of Tunis El Manar , Medical school of Tunis, 15 Djebel Lakhdhar Street La Rabta 1007 2-ENT department Rabta Hospital, La Rabta, Tunis1007, Tunisia. Sabrine Najjar; Resident Affiliations: 1-University of Tunis El Manar , Medical school of Tunis, 15 Djebel Lakhdhar Street La Rabta 1007. 2-ENT department Rabta Hospital, La Rabta, Tunis1007, Tunisia. Seif Boukriba ; MD Affiliations: 1-University of Tunis El Manar , Medical school of Tunis, 15 Djebel Lakhdhar Street La Rabta 1007 3-Radiology department Rabta Hospital, La Rabta, Tunis-1007, Tunisia . Baya Chelly; MD Affiliations: 1-University of Tunis El Manar, Medical school of Tunis, 15 Djebel Lakhdhar Street La Rabta 1007 4-Anatomopathology department Rabta Hospital, La Rabta, Tunis-1007, Tunisia . Houda Chahed; MD Affiliations: 1-University of Tunis El Manar, Medical school of Tunis, 15 Djebel Lakhdhar Street La Rabta 1007 2- ENT department Rabta Hospital, La Rabta, Tunis-1007, Tunisia. Ghazi Besbes; MD Affiliations: 1-University of Tunis El Manar , Medical school of Tunis, 15 Djebel Lakhdhar Street La Rabta 1007 2- ENT department Rabta Hospital, La Rabta, Tunis-1007, Tunisia.Corresponding Author:Azza Mediouni ; MD Professional Adress: Service d'ORL et de CCF, Hopital La Rabta, Tunis-1007, Tunisie. Tel number: 0021653938983 mail:azza.mediouni@gmail.comAbstract: The distinction between tuberculosis and sarcoidosis presents sometimes a clinical challenge. Their sequential occurrence in the same patient is uncommon. We present the case of a 42-year-old female with a proven diagnosis of tuberculous lymphadenitis, who has developed successively nasal tuberculosis and pulmonary and liver sarcoidosis. Key-words Granulomatous diseases, sarcoidosis, tuberculosis, association. Key Messages Tuberculosis and sarcoidosis are related in terms of pathogenesis and clinical manifestation, and may coexist in the same patient. When recurrent or resistant tuberculosis, one has to keep in mind a possible association to sarcoidosis.1. IntroductionTuberculosis and sarcoidosis are both chronic granulomatous diseases that display similarity in clinical presentation and histological features. Tuberculosis is an infection caused by Mycobacterium tuberculosis or bovis. Caseous necrosis is the pathologic hallmark of tuberculosis ${ }^{[1]}$. There are two forms of tuberculosis: active and latent. Latent tuberculosis is a state in which M. tuberculosis survives in the body without causing overt signs and symptoms ${ }^{[2]}$. The aetiology of sarcoidosis is still not settled. Though, the causal agent can be divided mainly into non-infectious and infectious causes. Several recent studies supported by evidence the hypothesis of mycobacterium role as a trigger antigen capable of inducing 
clinical expression of sarcoidosis ${ }^{[1]}$. Tuberculosis and sarcoidosis granulomatosis are sometimes difficult to separate, in the absence of a microbiological confirmation ${ }^{[2]}$. Nevertheless, ascertaining a precise diagnosis remains the key to management of these two diseases in view of different treatments. Three clinical associations of these two diseases have been reported in the literature: tuberculosis followed by sarcoidosis, co-existent sarcoidosis and tuberculosis and tuberculosis occurring in a chronic sarcoidosis ${ }^{[3]}$. The association between tuberculosis and sarcoidosis might remain complex and undetermined. The aim of this report is to critically evaluate the relationship between tuberculosis and sarcoidosis and expand further on the current debate.2. Case presentationA 42-year-old female patient with no medical history, presented with a chief complaint of bilateral neck masses three months ago. Physical examination revealed bilateral jugular nodes and left posterior nodes whose size was $1 \mathrm{~cm}$, firm, non tender and mobile. There was mucosal hypertrophy at the posterior wall of the rhinopharynx, which biopsy remained within the ordinary aspects. Tuberculin skin test showed no induration after 72 hours. Chest radiography was normal. Sputum sample for mycobacteria was negative. Fine needle aspiration cytology of the cervical lymphadenopathy has not been practised. An excision biopsy was taken and histopathological examination revealed necrotizing granulomatous inflammation suggestive of tuberculosis. The patient was treated with antituberculosis drugs (isoniazid $100 \mathrm{mg}$, rifampicin $900 \mathrm{mg}$, ethambutol 1,6g, and pyrazinamide $2 \mathrm{~g}$ : for two months followed by eight months of bitherapy) with a good compliance with treatment all along. The patient improved rapidly: enlarged cervical lymph nodes regressed remarkably. The initial histopathological findings in our patient supported the diagnosis of tuberculosis lymphadenitis, as mentioned above. This was further reconfirmed by resolution of patient's cervical enlarged lymph nodes with antituberculosis drugs. After 10 months of treatment, she again developed neck lymphadenopathy, with a recently associated nasal obstruction. Nasal endoscopy revealed mucosal hypertrophy with several nodules located on the nasal septum and inferior turbinate, whose biopsy concluded to nasal tuberculosis (figure 1). A regimen comprising isoniazid $100 \mathrm{mg}$, rifampicin 900mg and ethambutol $1200 \mathrm{mg}$ was reintroduced for six months daily, associated with ofloxacin $600 \mathrm{mg}$ for two months. Considering that the patient noticed some cough and arthralgia, the negatif tuberculin skin test, also subsequent occurrence of nasal tuberculosis and new lymphadenopathies in spite of antitubercular treatment, an associated sarcoidosis or VIH infection were suspected. Thoracic and abdominal CT scan was performed. It showed several nodular elements in both lungs (figure 2), with enlarged intra abdominal lymph nodes, liver micro-nodules and bilateral hilar and mediastinal adenopathy (figure 3). It appeared that the patient was non-reactive for human immunodeficiency virus (HIV). The patient was referred to the interventional pneumology department where bronchoalveolar lavage was practiced showing a lymphocytic alveolitis with a CD4/CD8 ratio of 5, and no acid-resistant bacilli were found in the lavage fluid. All these findings lead up to the diagnosis of pulmonary and hepatic sarcoidosis. Angiotensin-converting enzyme was elevated, and there was no sign of uveal involvement or hypercalcaemia. Introducing steroids treatment has been well discussed by the pneumologists, and it was not prescribed initially. After 18 months of treatment, cessation of antituberculosis drugs was decided seeing that the cervical lymphadenopathy showed progressive resolution and the nasal mucosa biopsy was negative. In view of cough exacerbation with recent dyspnea, and progressive deterioration shown in the CT scan consisting in pulmonary fibrosis appearance, and according to the lung function test results, corticosteroid treatment in form of oral prednisolone, $40 \mathrm{mg}$ daily, was introduced. The patient showed significant clinical improvement. After two months, the prednisolone was gradually tapered off and then completely stopped after about one year, when all the lesions cleared out. Her sarcoidosis remained quiescent on the most recent follow-up assessment after cessation of corticosteroid therapy.3. DiscussionSporadic case reports of sarcoidosis and tuberculosis association in the same patient have been reported in the literature documenting either a concomitant or sequential occurrence of sarcoidosis followed by tuberculosis or vice versa ${ }^{[4]}$. It's admitted that sarcoidosis treatment with corticosteroids or immunosuppressive drugs are risk factors for occurrence of mycobacterial infections during sarcoidosis. In a literature review, there were 27 well described case reports of Mycobacterium tuberculosis infection during sarcoidosis. Mycobacterial infections are third more frequent opportunistic infections happening in sarcoidosis patients (after aspergillosis and cryptococcosis) ${ }^{[5]}$. More than $66 \%$ of patients had extrapulmonary tuberculosis. An average time of three years after diagnosing sarcoïdosis was observed, and $74 \%$ were treated by corticosteroids or immunosuppressive drugs. Most of the cases (88\%) improved rapidly with 
antituberculosis drugs ${ }^{[5]}$. The immunological paradox of sarcoidosis; enhanced local immune response contrasting with peripheral CD4 lymphocytes anergy, is also responsible of opportunistic infections occurrence like tuberculosis. Considering this immunodepression, sarcoidosis can be revealed by tuberculosis or others opportunistic infections in patients not receiving immunosuppressive drugs ${ }^{[6]}$. In fact, tuberculin anergy is a clinically important phenomenon in sarcoidosis. As a negative tuberculin test excludes tuberculosis except in immunosuppressed individuals (as a latent sarcoidosis or co-infection with HIV), a great deal of importance must be attached to this test ${ }^{[7]}$. A bidirectional cohort study was recently published: its results strengthen the evidence that tuberculosis is a risk factor for sarcoidosis; patients with tuberculosis showed a higher risk of developing sarcoidosis than non-tuberculosis subjects. The highest risk of developing sarcoidosis was observed in patients with extrapulmonary tuberculosis. Sarcoidosis post-tuberculosis was likely to happen slowly, long after the tuberculosis has been treated ${ }^{[8]}$. An entity called "tuberculous sarcoidosis", associating clinical manifestations of both diseases have been described. Tuberculin test may be positive or negative, but the serum angiotensin converting enzyme levels are increased. CT of the chest reveals micro- or macronodules, with characteristic distribution in the peribronchovascular region and subpleural interstitium. The treatment involves combinations of steroids in large doses and antituberculosis drugs ${ }^{[9]}$. Agrawal and al postulate that tuberculosis and sarcoidosis may represent a spectrum of the same disease: they suggest that the difference in the immune response of the host may lead to varying degree of presentations: Sarcoidosis (S); Sarcoid-Tuberculosis (ST); Tuberculous- Sarcoid (TS) and Tuberculosis (TB) ${ }^{[1]}$. Evidence for the causal relationship between sarcoidosis and mycobacterial infection was firstly given by the presence of mycobacterial DNA in sarcoidosis tissue detected by polymerase chain reaction (PCR ) ${ }^{[10]}$. Recent studies considers that Mycobacterium tuberculosis indeed represents an important factor involved in the pathogenesis of sarcoidosis, that only some specific, poorly degraded mycobacterial pathogenic antigens contribute to immune-mediated granulomatous inflammation rather than the whole active mycobacteria and that they elicit a type IV immune response ${ }^{[11]}$. Dubaniewicz demonstrated, in a molecular analysis that not the whole M. tuberculosis cells but their particular antigens: hsp70Mtb, hsp65Mtb, and hsp16Mtb, could participate in the pathogenesis of sarcoidosis. The immunohistochemical analysis revealed their expression in all 25 lymph node tissues and tuberculoid granuloma from patients with sarcoidosis. The rest of the eight control cases were not hsp reactive ${ }^{[12]}$. However, a meta-analysis have demonstrated that mycobacterial antigens alone cannot explain the pathogenesis of this disease in all subjects, as no immune response to M. tuberculosis antigens or molecular evidence was observed in some sarcoidosis subjects. Therefore, it has been hypothesized that other factors besides mycobacterial antigens contribute to the pathogenesis of sarcoidosis ${ }^{[11]}$. The classic manifestations of tuberculosis and sarcoidosis have an overlapping range for which it is sometimes difficult to make a clinical diagnosis ${ }^{[13]}$. Although it is a significant global challenge, sarcoidosis is increasingly being diagnosed in countries with a high tuberculosis burden. This is due to increased awareness and better availability of diagnostic modalities especially microbiology and histopathology ${ }^{[2]}$. Many authors suggest that tuberculosis can flare-up with the administration of corticosteroids, so that tuberculosis prevention must be systematic in high risk TB patients requiring corticosteroids. An antituberculous chemoprophylaxis must be introduced over one month before administrating TNF inhibitors ${ }^{[5]}$. Even though our observation belongs to the gray zone of tuberculous sarcoidosis spectrum, the question remains whether the etiologic agent of sarcoidosis in our patient was mycobacterial antigens; or was tuberculosis subsequent to sarcoidosis induced immune depression?4. ConclusionThis case report demonstrates that when recurrent or resistant tuberculosis is noticed, one has to keep in mind possible association to sarcoidosis. As tuberculosis and sarcoidosis are closely related in terms of pathogenesis and clinical manifestation, and may even coexist in the same patient, the diagnosis may be challenging especially in endemic tuberculosis zone.Contribution Details:Azza Mediouni: Definition of intellectual content, Data analysis, Literature search, Draft Revision, Manuscript preparation. Sabrine Najjar: Data acquisition, Literature search, Data analysis, drafting manuscript. Seif Boukriba: Data analysis Baya Chelly: Data analysis Chahed Houda: Revising critically the draft. Ghazi Besbes : Revising critically the draft.5. References1. Agrawal R, Kee AR, Ang L, Tun Hang Y, Gupta V, Kon OM, et al. Tuberculosis or sarcoidosis: Opposite ends of the same disease spectrum? Tuberculosis. 2016; 98:21-6. 2. Mortaz E, Masjedi MR, Matroodi S, Abedini A, Kiani A, Soroush D, et al. Concomitant patterns of tuberculosis and sarcoidosis. Tanaffos. 2013; 12(4): 6 3. Shah JR. Tberculous sar- 
coidosis. Lung India. 2007; 24 : 83-86 4. Mohapatra PR, Garg K, Singhal N, Aggarwal D, Gupta R, Khurana A, et al. Lymphadénite tuberculeuse dans un cas de sarcoidose bien gérée. Indian J Chest Dis Allied Sci. 2013; 55: 217-20. 5. Jamilloux Y, Bernard C, Lortholary O, Kerever S, Lelievre L, Gerfaud-Valentin M, et al. Les infections opportunistes au cours de la sarcoïdose. Rev Med Interne. 2016; 38(5): 320-27. 6. Didier K, Servettaz A, Tabary T, Nguyen Y, Bani-Sadr F. Deux infections opportunistes successives associées à une lymphopénie CD4 sévère révélant une sarcoïdose systémique. Presse Med. 2018; 47(3): 296-97. 7. Babu K. Sarcoidosis in tuberculosis-endemic regions: India. J Ophtalmic Inflamm Infect. 2013; 3(1):53. 8. Wang SH, Chung CH, Huang TW, Tsai WC, Peng CK, Huang KL, et al. Bidirectional association between tuberculosis and sarcoidosis. Respirology. 2019. 9. Sureka B, Bansal K, Arora A. Tubercular Sarcoidosis: An intriguing concoction of tuberculosis and sarcoidosis. AJR American journal of roentgenology. 2015; 205(2):W229. 10. Wong CF, Yew WW, Wong PC, Lee J. A case of concomitant tuberculosis and sarcoidosis with mycobacterial DNA present in the sarcoid lesion. Chest. 1998; 114(2):626-9. 11. Fang C, Huang H, Xu Z. Immunological Evidence for the Role of Mycobacteria in Sarcoidosis: A Meta-Analysis. PloS one. 2016; 11(8):e0154716. 12. Dubaniewicz A, Dubaniewicz-Wybieralska M, Sternau A, Zwolska Z, Izycka-Swieszewska E, AugustynowiczKopec E, et al. Mycobacterium tuberculosis complex and mycobacterial heat shock proteins in lymph node tissue from patients with pulmonary sarcoidosis. Journal of Clinical Microbiology. 2006; 44(9):3448-51. 13. Carbonelli C, Giuffreda E, Palmiotti A, Loizzi D, Lococo F, Carpagnano E, et al. Coexistent sarcoidosis and tuberculosis: a case report. Respiration. 2017; 93(4):296-300.Legends for Figures Figure 1: Hematoxylin and eosin staining of nasal mucosa showing tuberculous granuloma with central caseous necrosis. Figure 2: Coronal thoracic CT scan showing nodular diffuse ground-glass lesions with peri bronchio-vascular and sub pleural distribution. Figure 3: Coronal cervico-thoraco-abdominal CT scan showing multiples cervical, mediastinal and abdominal non necrotised adenopathies, associated to hypo dense micro-nodular lesions of liver.

\section{Hosted file}

images page.docx available at https://authorea.com/users/417521/articles/539115-head-andneck-tuberculosis-associated-to-sarcoidosis-a-case-report 